\title{
RELEVÂNCIA DE DOIS FATORES NA PERCEPÇÃO DE QUALIDADE EM SACS
}

\section{Adele Queiroz}

Mestranda em Administração de Empresas pela EAESP/FGV.

E-mail: adele@uol.com.br

Fernando Natal de Pretto

Mestrando em Administração de Empresas pela EAESP/FGV.

Gustavo Corrêa Mirapalheta

Doutorando em Administração de Empresas pela EAESP/FGV.

\section{RESUMO}

Este artigo defende a hipótese de que a qualidade percebida pelo consumidor no serviço prestado pelos Serviços de Atendimento ao Consumidor (SACs) no atendimento telefônico de reclamações é mais influenciada pela percepção da qualidade do atendimento do que pela resolução do problema que gerou a reclamação. 0 trabalho apresenta uma análise teórica dos processos que levam o consumidor à reclamação e os resultados de uma pesquisa de campo realizada para testar a hipótese proposta em uma empresa de grande porte do setor eletroeletrônico com operações no Brasil.

\section{ABSTRACT}

This article analyses the quality perceived by the consumer in the customer services offered by SACs. It defends the hypothesis that the perceived quality is more influenced by the perception of the quality of the assistance itself than by the solution of the problem that caused the complaint. This text presents a theoretical analysis of the processes that drive the consumer to complain and the results of an empirical research conducted to test the hypothesis in a large company of the electric-electronic sector in Brazil.

\section{PALAVRAS-CHAVE}

Reclamações, atendimento telefônico, SACs, qualidade percebida, comportamento do consumidor.

\section{KEY WORDS}

Complaints, call centers, customer services, perceived quality, consumer behavior. 


\section{O MODELO DE RECLAMAÇÃO DE HIRSCHMAN}

A literatura referente à análise do comportamento do consumidor e sua percepção da qualidade no caso de Serviços de Atendimento ao Consumidor (SACs) é um campo pouco explorado. Isso é interessante se levarmos em conta que os modelos mais antigos que procuram descrever o comportamento do consumidor em situações de descontentamento datam do início da década de 70 , e a descrição dos processos que levam até a reclamação é encontrada na década de 80 (Singh e Wilkes, 1996).

Considerando-se que os SACs podem ser utilizados como um excelente canal de comunicação com o mercado, a aplicação de tal modelagem mostra-se muito importante para os resultados da empresa. Apesar de não ser essa a finalidade do presente artigo, vale a pena ressaltar que um entendimento mais profundo das causas de uma reclamação, as quais não são necessariamente esclarecidas pelo próprio cliente que se manifesta de tal forma (Bagozzi, 1982), pode levar a uma melhoria na compreensão das necessidades dos consumidores e mesmo a um aumento na competitividade da empresa.

Conforme mostra Hirschman (1970), as formas de manifestação de uma insatisfação podem ser classificadas em três tipos: Voz, Saída e Propaganda Boca-a-Boca Negativa ${ }^{1}$. Seu modelo apresenta a reclamação como o último nível de um processo que depende de fatores situados em três níveis (ver Figura 1).

Figura 1 - Modelo de Reclamação de Hirschman

\begin{tabular}{|c|}
\hline $\begin{array}{c}\text { Modelo de Hirschman para a Reclamação } \\
\text { Nível 1: Manifestação da reclamação } \\
\text { Voz, saída e propaganda negativa }\end{array}$ \\
\hline Nível 2: Características do indivíduo \\
Avaliação cognitiva \\
Percepção de valor agregado \\
Sofisticação \\
\hline Nível 3: Características da indústria \\
Concentrada \\
Competitiva \\
Oligopólio competitivo
\end{tabular}

Segundo esse modelo, se a avaliação cognitiva for positiva, ou seja, se houver uma probabilidade percebida de se obter sucesso com a reclamação, o consumidor se manifestará desta forma. Se ocorreram casos anteriores de sucesso com o consumidor ou se ele tiver conhecimento de tais casos, mais forte será a intenção de reclamar. Por último, o mesmo estudo mostrou uma influência positiva de fatores de formação na probabilidade de o consumidor se manifestar com uma reclamação.

O que é interessante em tal estudo é o aparecimento explícito de características macroambientais que influenciam de forma decisiva a opção por uma reclamação. Essas características são determinadas essencialmente pelo modelo de competição da indústria. Em situações que o autor chama de "concentradas" (por exemplo, nos monopólios), os clientes optariam por reclamar (voz) e, no caso de indústrias em ambientes "competitivos", por trocar de produto (saída).

Para o presente estudo, a situação de maior interesse é a do oligopólio competitivo. Hirschman concentra sua análise nessa situação (já que é a mais freqüente em sociedades industrializadas, como a brasileira), análise essa validada por Andreasen (1985), que conduz um estudo acerca das teorias de Hirschman sobre oligopólios competitivos.

\section{O MODELO DE AÇÃO RACIONAL DE FISHBEIN/AJZEN APLICADO ÀS CAUSAS DE UMA RECLAMAÇÃO}

Após determinar as ações possíveis e prováveis que um consumidor pode tomar, em especial no caso de oligopólios competitivos, surge a questão: quais fatores motivariam tais atitudes? Para o estudo destes, lança-se mão do modelo de Fishbein/Ajzen (1975), que prediz que "uma medida de intenção comportamental medirá o desempenho de qualquer ato voluntário, a menos que a intenção mude antes do desempenho ou a medida utilizada não possua correspondência com o critério comportamental em termos de ação, objetivo, contexto, escala temporal e/ou especificidade".

Esse modelo (já clássico, como pode ser facilmente verificado pelas extensas citações referentes a ele na literatura) sofreu, ao longo dos anos, uma série de melhorias ${ }^{2}$ que, no entanto, não alteraram sua proposição básica: a importância da medida de intenção comportamental. 
Para o caso em estudo, as contribuições mais importantes vêm de dois autores.

O primeiro autor é Sheppard (1988), que mostrou a necessidade de se incluir no modelo original:

a) a diferenciação entre intenção (comportamento passível de ser realizado de imediato) e objetivo (comportamento que demanda planejamento, como, por exemplo, a compra de uma casa);

b) a possibilidade de o consumidor ser exposto a uma série de alternativas com probabilidades iguais de concretização.

No caso da diferenciação entre intenção e objetivo, todas as reclamações seriam classificadas como intenções, já que o consumidor encontra pouca ou nenhuma dificuldade para fazer uma reclamação, seja ela telefônica ou por escrito. $\mathrm{O}$ que merece atenção é a questão das opções, pois, no modelo de Hirschman, o consumidor termina com três opções e sua decisão de manifestação por meio dos SACs, em termos teóricos, seria representada por uma saída pela opção "voz".

O segundo autor é Dabholkar (1994), que realizou um estudo de incorporação do fator possibilidade de escolha ao modelo de Fishbein/ Ajzen. O modelo de Dabholkar assume que o consumidor que irá tomar uma decisão tem muitas intenções em mente antes de escolher uma alternativa. $\mathrm{O}$ modelo é adequado a situações em que há um considerável espaço de tempo entre intenções e comportamentos e nas quais o indivíduo pode formar intenções sob a forma de "estimativas" de engajamento em alternativas de comportamentos futuros. Essas alternativas podem ser concebidas em momentos diferentes e, na ocasião da tomada de decisão, elas podem todas ser comparadas.

\section{A CAUSA INICIAL: AS REAÇÕES DO CONSUMIDOR A UMA FALHA EM UM PRODUTO - O SISTEMA ATRIBUICIONAL}

Por fim, chega-se ao ponto de modelar a insatisfação no momento de sua ocorrência, como, por exemplo, quando ocorre uma falha em um produto e/ou um erro em algum sistema de atendimento. Para tanto, lança-se mão do chamado modelo atribuicional (Folkes, 1984). Esse modelo procura relacionar pensamentos específicos a respeito de falhas nos produtos com comportamentos relacionados a reclamações.
Nesse contexto, as pessoas são vistas como processadores de informações racionais, cujas ações são influenciadas por suas inferências a respeito de possíveis causas na falha de um produto. Indo além, as pessoas procuram determinar o porquê da falha, e o tipo de explicação encontrada determina o curso de ação a ser tomado.

\section{As pessoas procuram determinar 0 porquê da fallha, e o tipo de explicação encontrada determina o curso de ação} a ser tomado.

As explicações podem localizar-se em três níveis, de acordo com a estabilidade (se o problema é persistente ou não), a localidade (se o agente causador foi a empresa ou o consumidor) e a controlabilidade (se as causas podem ser determinadas e resolvidas ou não).

Desses três fatores, a localidade é o que mais leva o consumidor a manifestar-se por meio da "voz" (especialmente em situações de monopólio competitivo), seguida de perto por combinações variadas dos outros dois fatores com a localidade.

Com isso, conclui-se o modelo teórico aqui apresentado para o processo de reclamação. $\mathrm{O}$ consumidor que procura um SAC, de acordo com o modelo, tem as seguintes características: a) decidiu manifestar-se por meio de uma reclamação formal em vez de realizar a troca de marca ou partir para a propaganda negativa boca-a-boca;

b) está inserido em um contexto de oligopólio competitivo;

c) teve uma "intenção";

d) a sua intenção foi gerada por uma inferência a respeito da causa possível da falha do produto. $\mathrm{O}$ que se procura testar a partir de agora é como uma pessoa inserida em tal situação percebe a qualidade no Serviço de Atendimento ao Consumidor. Mais especificamente, se o peso maior para a qualidade percebida encontra-se na resolução efetiva do problema (nesse caso, os fatores determinantes dessa percepção seriam atribuicionais) ou na forma do atendi- 
mento (e, nesse caso, os fatores de maior peso seriam contextuais).

\section{METODOLOGIA}

O trabalho de campo foi realizado com uma amostra de 78 clientes que recorreram ao SAC de uma empresa de grande porte com operações no Brasil, do setor eletroeletrônico, ora denominada "Empresa X". Foram utilizados casos de reclamações que ocorreram entre $1^{\circ}$ de março de 1997 e 27 de maio de 1997 e que foram considerados pela empresa como solucionados. Os consumidores pesquisados vivem na Grande São Paulo.

Considerou-se que a empresa atua num ambiente de oligopólio competitivo, e a escolha dessa empresa especificamente deveu-se:

a) ao fato de produzir e comercializar bens de consumo de alto valor unitário, cujas manifestações de reclamação relativas a esses produtos podem ser mais incisivas do que no caso de produtos de baixo valor unitário;

b) ao fato de seu SAC ser constituído por uma equipe que passa por constantes programas de treinamento e desenvolvimento.

A pesquisa procurou analisar o peso relativo da resolução efetiva do problema de acordo com as expectativas do cliente versus a percepção do cliente em relação à qualidade no atendimento recebido ao manifestar-se por meio de uma reclamação.

Esses dois fatores - resolução do problema e forma do atendimento - foram escolhidos a partir dos resultados de uma pesquisa qualitativa encomendada anteriormente pela própria organização, cujos resultados indicaram terem sido os dois únicos que se destacaram intensamente entre os fatores que representam expectativas dos consumidores. Pelos resultados da pesquisa qualitativa, esses dois fatores situaram-se bem próximos na percepção de qualidade do consumidor, tendo sido considerados com grau de importância semelhante. $\mathrm{O}$ trabalho citado foi rea- lizado por uma empresa de reconhecida competência no mercado e que se utiliza das boas normas de pesquisa. Por questões de sigilo, a empresa pesquisada permitiu o acesso aos resultados, porém solicitou que os nomes não fossem divulgados.

$\mathrm{O}$ instrumento da presente pesquisa foi baseado no método de questionário aplicado por telefone, seguindo a metodologia descritiva (Aaker, Kumar e Day, 1995). Inicialmente, o consumidor foi informado de que estava sendo realizada uma pesquisa para a "Empresa X", que forneceu a ele um equipamento $\mathrm{Y}$, cuja reclamação havia sido cadastrada na data Z. Foi então solicitado a contribuir para esse trabalho, respondendo a quatro questões:

1. A solução apresentada para o seu caso deixou-o satisfeito?
( ) $\operatorname{sim}$
( ) não

2. O Sr./Sra. se sentiu bem atendido pelo SAC da "Empresa X"?
( ) $\operatorname{sim}$
( ) não

3. Se o Sr./Sra. tivesse que dar uma nota para o SAC da "Empresa X", que nota daria?

(0) (1) (2) (3) (4) (5) (6) (7) (8) (9) (10)

4. O Sr./Sra. compraria novamente um produto da "Empresa X"?
( ) $\operatorname{sim}$
( ) não

\section{ANÁLISE DOS DADOS EMPÍRICOS}

Nos resultados da aplicação do questionário, as notas resultantes da terceira questão foram agrupadas segundo as respostas apresentadas às duas primeiras questões. A quarta pergunta foi tabulada de acordo como o percentual de respostas "sim" dentro do grupo.

Foram, em seguida, realizados dois testes estatísticos de duas médias com dados desemparelhados e amostra pequena (Aczell, 1993), para determinar o quanto a variação individual das respostas afeta a nota dada ao SAC.

Os resultados são apresentados na Tabela 1.

Tabela 1 - Resultados das respostas ao questionário

\begin{tabular}{|c|c|c|c|c|}
\hline $\begin{array}{c}\text { Respostas } \\
\text { às perguntas 1 e 2 }\end{array}$ & $\begin{array}{c}\text { Nota } \\
\text { média }\end{array}$ & $\begin{array}{c}\text { Desvio-padrão } \\
\text { (amostra) }\end{array}$ & $\begin{array}{c}\text { № de } \\
\text { respondentes }\end{array}$ & $\begin{array}{c}\text { Recompra } \\
\text { (em \%) }\end{array}$ \\
\hline S/S & 9,33 & 1,09 & 36 & 94 \\
\hline N/S & 7,80 & 1,50 & 20 & 75 \\
\hline S/N & 4,00 & NC & 1 & NC \\
\hline N/N & 2,66 & 2,26 & 21 & 28 \\
\hline
\end{tabular}


O primeiro teste realizado considerou a comparação de duas médias com as seguintes hipóteses:

$\mathrm{H}_{0}$ - Os dois grupos de pessoas, os quais originaram as amostras dos que responderam $\mathrm{S} / \mathrm{S}$ e $\mathrm{N} / \mathrm{S}$, não se diferenciam quanto às notas do atendimento no SAC, ou seja, a média das notas dos dois grupos é igual.

$\mathrm{H}_{1}$ - Há diferença entre os dois grupos e, ainda, a média dos que responderam $\mathrm{S} / \mathrm{S}$ é maior que a dos que responderam N/S.

Com base nos dados da Tabela 1 , foi calculado o valor experimental " $\mathrm{t}$ ” (Student), com o resultado apresentado no Quadro 1.

Quadro 1 - Primeiro teste: comparação entre S/S e N/S

$\mathrm{t}$ (experimental)

Pode-se dizer, portanto, que existe evidência para

afirmar, com $99 \%$ de certeza, que a média da resposta S/S é maior que a média da resposta N/S.

O segundo teste realizado também considerou a comparação de duas médias com as seguintes hipóteses:

$\mathrm{H}_{0}$ - Os dois grupos de pessoas, os quais originaram as amostras dos que responderam N/S e N/N, não se diferenciam quanto às notas do atendimento no SAC, ou seja, a média das notas dos dois grupos é igual.

$\mathrm{H}_{1}$ - Há diferença entre os dois grupos e, ainda, a média dos que responderam N/S é maior que a dos que responderam N/N.

Com base nos dados da Tabela 1 , foi calculado o valor experimental " $\mathrm{t}$ " (Student), obtendo-se o resultado apresentado no Quadro 2.

Quadro 2 - Segundo teste: comparação entre N/S e N/N

\begin{tabular}{l}
\hline $\mathrm{t}$ (experimental) \\
Pode-se dizer, portanto, que existe evidência para \\
afirmar, com 99\% de certeza, que a média da \\
resposta N/S é maior que a média da resposta N/N.
\end{tabular}

Não existiram dados estatisticamente significativos para realizar o teste com o par de respostas $\mathrm{S} / \mathrm{N}$.

Se considerarmos as situações em que as respostas às perguntas 1 e 2 foram $\mathrm{S} / \mathrm{S}$ (ou seja, quando o consumidor mostrou-se satisfeito com a solução dada para o seu problema e também com o atendimento que recebeu do SAC), temos que a média das notas dadas para o SAC foi 9,33 (num intervalo de 0 a 10).
Nas situações em que as respostas para essas mesmas perguntas foram N/S (quando o consumidor não ficou satisfeito com a solução dada para o seu problema, mas ficou satisfeito com o atendimento que recebeu do SAC), a média das notas do SAC foi 7,80 .

\section{0 peso maior para a qualidade percebida encontra-se na resolução efetiva do problema ou na forma do atendimento?}

Podemos afirmar também que, nessas duas situações em que o consumidor se mostra satisfeito com a forma como foi atendido pelo SAC, a média das notas dadas pelos consumidores que não estão satisfeitos com a solução dada para problema foi 7,80 , então, com $99 \%$ de certeza, a média das notas dadas por aqueles que estão também satisfeitos com a solução do problema é superior quando se consideram os dados colhidos, no caso, 9,33.

Por outro lado, nas situações em que as respostas dos consumidores às duas primeiras perguntas foram N/N (ou seja, não estavam satisfeitos com a solução do problema nem com o atendimento que receberam do SAC), a média das notas foi 2,66 .

Se compararmos com a outra situação em que também os consumidores não estavam satisfeitos com o atendimento que receberam $(\mathrm{S} / \mathrm{N})$, temos que, se a média das notas dadas pelos consumidores que não estavam satisfeitos com a resolução do problema nem com o atendimento recebido é 2,66, então, com $99 \%$ de certeza, a média das notas daqueles que não estavam satisfeitos com a solução, mas sim com o atendimento recebido, é superior, sempre considerando os dados colhidos, no caso, 7,80.

\section{DISCUSSÃO}

Percebe-se claramente que, apesar de existir evidência estatística de que a resolução do problema de acordo com a expectativa do consumidor influencia a nota atribuída à sua percepção de qualidade em relação ao SAC, o esteio principal para a nota atribuída ao SAC, entre os 
dois fatores considerados, não é a resolução do problema de acordo com as expectativas do consumidor, mas sim a sua percepção de que recebeu um bom atendimento.

A análise das médias das notas mostra uma clara mudança entre as situações em que o consumidor está ou não satisfeito com o atendimento, pois, no primeiro caso, as médias são 9,33 e 7,80 (respectivamente para os casos em que os clientes estavam ou não satisfeitos também com a resolução do problema) e, no segundo caso, a média cai para 2,66 (não satisfeitos com o atendimento nem com a solução do problema).

Isso é reforçado, ainda, pelo fato de não existirem na amostra quantidades estatisticamente significativas de consumidores que julgaram ter o problema resolvido da maneira que esperavam e ao mesmo tempo não se julgaram bem atendidos, ou seja, entre os dois fatores considerados, o maior peso na percepção de qualidade no SAC pelo cliente é proveniente de um bom atendimento.

\section{LIMITAÇÕES}

De acordo com o modelo de Hirschman, um dos fatores determinantes da reclamação é o grau de sofisticação pessoal do indivíduo. $\mathrm{O}$ presente estudo não levou em conta esse tipo de estratificação, considerando apenas, de acordo com o mesmo modelo, a estrutura do mer- cado no qual está inserido, ou seja, um oligopólio competitivo.

Outra limitação diz respeito ao espaço temporal no qual foi tomada a amostra, que, por ser pequeno (cerca de três meses), expõe o estudo a possíveis variações sazonais do mercado, também não consideradas neste trabalho.

\section{ESTUDOS FUTUROS}

Aparentemente, a amostra analisada apresentou uma correlação entre o percentual de clientes que comprariam novamente produtos dessa marca (lealdade à marca) e a nota média atribuída ao Serviço de Atendimento ao Consumidor. Não será feita uma análise das implicações de tal resultado neste trabalho, tendo ele sido apresentado apenas para fins de pesquisas futuras.

Sabe-se que, no setor de eletroeletrônicos, o fator sazonalidade é importante, e estudar o quanto esse fator provoca variações nas médias obtidas seria outra área de pesquisa.

Finalmente, um interessante estudo seria replicar esta pesquisa somente com manifestações de reclamações reincidentes, ou seja, com consumidores cujo produto que gerou a reclamação já havia apresentado problemas anteriormente, administrados pelo SAC. Poder-se-ia checar, assim, se a importância dos fatores estudados no presente trabalho na percepção de qualidade do SAC pelo consumidor se alteraria dentro do novo contexto analisado. $\bigcirc$

\section{REFERÊNCIAS BIBLIOGRÁFICAS}

AAKER, David A., KUMAR, V., DAY, George S. Marketing research. New York: John Wiley \& Sons, 1995.

ACZELL, Amir D. Complete business statistics. Boston, MA Richard D. Irwin Inc., 1993.

ANDREASEN, Alan R. Consumer responses to dissatisfaction in loose monopolies. Journal of Consumer Research, v.12, Sept. 1985

BAGOZZI, Richard P. A field investigation of causal relations among cognitions, affect, intentions, and behavior. Journal of Marketing Research, v.19, p.562-84, Nov. 1982.
DABHOLKAR, Pratibha A. Incorporating choice into an attitudinal framework: analysing models of mental comparison processes. Journal of Consumer Research, v.21, June 1994.

FISHBEIN, Martin, AJZEN, Icek. Belief, attitude, intention and behavior. an introduction to theory and research. MA: AddisonWesley, 1975.

FOLKES, Valerie S. Consumer reactions to product failure: an attribuitional approach. Journal of Consumer Research, v.10, Mar. 1984.
HIRSCHMAN, Albert 0. Exit, voice and loyalty. responses to decline in firms, organizations and states. Cambridge: Harvard University Press, 1970.

SHEPPARD, Blair H., HARTWICK, Jon, WARSHAW, Paul R. The theory of reasoned action: a meta-analysis of past research with recommendations for modifications and future research. Journal of Consumer Research, v.15, Dec. 1988.

SINGH, Jagdip, WILKES, Robert E. When customers complain: a path analysis of the key antecedents of consumer complaint responses estimates. Journal of the Academy of Marketing Science, v.24, n.4, p.350-65, 1996

\section{NOTAS}

Os autores gostariam de agradecer especialmente a colaboração do Prof. Rubens da Costa Santos.

1. "Voice, exit and negative word-of-mouth", no original em inglês.
2. Ver BAGOZZI, Richard P. A field investigation of causal relations J. An experimental investigation of causal relations among among cognitions, affect, intentions, and behavior. Journal of cognitions, affect, and behavioral intentions. Journal of Consumer Marketing Research, v.19, p.562-84, Nov. 1982 e LUTZ, Richard Research, v.3, n.4, p.197-208, Mar. 1977. 